\title{
Nanomechanical properties of solvent cast polystyrene and poly(methyl methacrylate) polymer blends and self-assembled block copolymers
}

\author{
Matteo Lorenzoni, ${ }^{\mathrm{a}, *}$ Laura Evangelio, ${ }^{\mathrm{a}}$ Célia Nicolet, ${ }^{\mathrm{b}}$ Christophe Navarro, ${ }^{\mathrm{b}}$ Alvaro San Paulo,, \\ Gemma Rius, ${ }^{d}$ and Francesc Pérez-Murano ${ }^{a}$ \\ ${ }^{a}$ Instituto de Microelectrónica de Barcelona (IMB-CNM, CSIC) Campus de la UAB, 08193 Bellaterra, Spain \\ ${ }^{\mathrm{b}}$ Arkema France, R.N. 117, BP34-64170 Lacq, France \\ 'Instituto de Microelectrónica de Madrid (CSIC) Calle de Isaac Newton, 8, 28760 Tres Cantos, Spain \\ ${ }^{d}$ Nagoya Institute of Technology, NITech, Gokiso, Showa, 466-8555 Nagoya, Japan
}

\begin{abstract}
The nanomechanical properties of solvent-cast polymer thin films have been investigated using PeakForce $^{\mathrm{TM}}$ Quantitative Nanomechanical Mapping. The samples consisted of films of polystyrene (PS) and poly(methyl methacrylate) (PMMA) obtained after the dewetting of toluene solution on a polymeric brush layer. Additionally, we have probed the mechanical properties of poly(styrene-b-methyl methacrylate) block copolymers (BCP) as randomly oriented thin films. The probed films have a critical thickness $<50 \mathrm{~nm}$ and present features to be resolved $<42 \mathrm{~nm}$. The Young's modulus values obtained through several nanoindentation experiments present a good agreement with previous literature, suggesting that the PeakForce ${ }^{\mathrm{TM}}$ technique could be crucial for BCP investigations, e.g., as a predictor of the mechanical stability of the different phases. @ 2015 Society of Photo-Optical Instrumentation Engineers (SPIE) [DOI: 10.1117/1.JMM.14.3.033509]
\end{abstract}

Keywords: nanomechanical properties; PeakForce ${ }^{\mathrm{TM}}$; block copolymer.

Paper 15021P received Mar. 16, 2015; accepted for publication Jul. 24, 2015; published online Sep. 11, 2015.

\section{Introduction}

Direct self-assembly (DSA) of block copolymer (BCP) is becoming a well-established alternative patterning method for the creation of regular arrays ${ }^{1,2}$ that could potentially overcome the sub-10 $\mathrm{nm}$ resolution limit. ${ }^{3}$ Based on their high throughput and relatively simple processing, DSA-BCP thin films are likely to be adopted by the microelectronics industry within a few years. BCPs are macromolecules that are formed by two (or more) distinct polymer chains (blocks) joined by interblock covalent bonds. The two phases represented by the blocks, which are thermodynamically incompatible, tend to segregate according to a balance between repulsive and attractive intermolecular forces. Phase separation leads to a regular arrangement in different structural configurations (lamellae, cylinders, spheres, or other more complex shapes) depending on several parameters, mainly the ratio of the molecular weights of the blocks forming the copolymer. ${ }^{4}$

A special focus has been put on measuring the mechanical properties of such phases once the segregation in lamellaelike configuration has occurred. Access to the mechanical characteristics of nanodomains both over the surface and across the film (three-dimensional morphology) represent an important source of information that could explain the nature of morphological defects, reveal the presence of wetting layers, and identify selective hardening due to aging. ${ }^{5}$

Atomic force microscopy (AFM) can overcome the limitations usually associated with nanoindentation i.e., the probe size and the minimum indentation depth required $(>10 \mathrm{~nm})$. AFM is widely used to simultaneously reproduce

*Address all correspondence to: Matteo Lorenzoni, E-mail: matteo.lorenzoni@ imb-cnm.csic.es surface topography at the nanometric scale and map qualitative differences of local surface properties, such as friction, adhesion, or elastic modulus. ${ }^{5,6}$ For example, using PeakForce ${ }^{\mathrm{TM}}$ Quantitative Nanomechanical Mapping $\left(\mathrm{QNM}^{\mathrm{TM}}\right)$, it is possible to reliably measure Young's modulus of materials with high spatial resolution and surface sensitivity. ${ }^{7,8}$ Basically, with this technique, it is possible to acquire multiple force distance curves with improved force resolution, i.e., obtaining real-time calculation of mechanical properties at each tip-surface contact. However, the method is particularly challenged when the material to be indented is deposited on a rigid substrate and reaches certain critical thicknesses in the same order of magnitude of the induced/applied mechanical deformation.

In this work, we apply PeakForce ${ }^{\mathrm{TM}} \mathrm{QNM}^{\mathrm{TM}}$ technique to characterize the mechanical properties of polymer thin film resists and DSA-BCPs toward optimizing their processing and technology of resist materials for advanced lithography and pattern transfer. First, we have mapped and identified the formation of different phases after dewetting of polystyrene (PS) and poly(methyl methacrylate) (PMMA) homopolymer on a substrate that presents distinct affinity with the different components. The substrate is a PS brush layer, which is usually employed as a preferential brush layer for BCP alignment. With these preliminary experiments, we determine the proper indentation conditions for the two materials we are investigating. Moreover, it allows us to verify that thin films of such polymers can be successfully probed and characterized by PeakForce ${ }^{\mathrm{TM}}$. In fact, for thicknesses $<50 \mathrm{~nm}$, the tip senses the substrate even for very small loads so that the mechanical properties are dominated by the stiff substrate, resulting in rather large apparent modulus values compared

$1932-5150 / 2015 / \$ 25.00$ @ 2015 SPIE 
to the bulk one. ${ }^{9}$ It is known that when the indentation depth exceeds $20 \%$ of the thickness of the probed film, effects of the substrate underneath should be considered. ${ }^{10}$ Second, we have probed the mechanical properties of randomly oriented poly(styrene-b-methylmethacrylate) (PS-b-PMMA) thin films. Thin films of PS-b-PMMA can self-assemble into ordered periodic structures at the molecular scale $(5$ to $50 \mathrm{~nm})$, forming a rich variety of nanophase-separated structures such as lamellae or pillars. PeakForce ${ }^{\mathrm{TM}}$ based AFM nanoindentation provides important information regarding differences in the mechanical properties of the dissimilar oriented domains by probing a few nanometers underneath the surface, thus being able to overcome typical limitations of surface analysis (as in the case of friction maps). For instance, it is likely that the mechanical stress introduced with orientation could eventually be measured by local changes in the stiffness of the different phases of the BCP differentiating between ordered and randomly oriented regions. Generally speaking, PeakForce $^{\mathrm{TM}}$ tapping AFM should be adopted for BCP characterization because it (1) interacts with nanometer size domains, returning quantitative information regarding a very small volume (i.e., PMMA or PS single domains) ${ }^{11}$ and (2) is able to provide a large amount of data for submicrometric areas. The purpose of this work is to show the great potential of the technique when applied to BCP thin films. In terms of lateral resolution, probing nanometer-long structures, like the ones created by the self-assembly of BCPs, brings the technique to its spatial limits. ${ }^{12}$

\section{Materials and Methods}

\subsection{Grafting of the Hydroxyl-Terminated Polystyrene Brush Layer}

The rigid substrates are p-type silicon ( 4 to $40 \Omega \cdot \mathrm{cm}$ resistivity) chips bearing a native silicon oxide layer. The brush layer is obtained from hydroxyl-terminated polystyrene (PS-OH) (number average molecular weight $(\mathrm{Mn})=4.5 \mathrm{~kg} \cdot \mathrm{mol}^{-1}$, polydispersity index $(\mathrm{PDI})=1.09$ ), purchased from Polymer Source, Inc. The grafting process starts by coating the silicon surface with the polymer brush. Previously, the silicon surface has been cleaned and activated by $\mathrm{O}_{2}$ plasma for $10 \mathrm{~min}$ to favor the reaction between the hydroxyl groups from the PS-OH and the native oxide of the substrate. A $40 \mathrm{~nm}$ thick PS-OH brush layer is deposited by spin coating at $5000 \mathrm{rpm}$ from a $1.5 \%(\mathrm{w} / \mathrm{w})$ toluene solution. Subsequently, samples are annealed in a nitrogen environment at $260^{\circ} \mathrm{C}$ for $5 \mathrm{~min}$. After the annealing, the unreacted $\mathrm{PS}-\mathrm{OH}$ is rinsed away with toluene by ultrasonication at $40^{\circ} \mathrm{C}$ for 5 min leaving a grafted PS layer of $\sim 5 \mathrm{~nm}$.

\subsection{Homopolymer Films and Block Copolymer Self-Assembly}

Homopolymers employed are PS $\left(\mathrm{Mn}=38.6 \mathrm{~kg} \cdot \mathrm{mol}^{-1}\right.$, $\mathrm{PDI}=1.12)$ and PMMA $\left(\mathrm{Mn}=38.6 \mathrm{~kg} \cdot \mathrm{mol}^{-1}\right.$, PDI $=$ 1.12). of the homopolymers propylene glycol methyl ether acetate (PGMEA) solutions are spin-coated at $2500 \mathrm{rpm}$ for $30 \mathrm{~s}$ forming a film of $\sim 20 \mathrm{~nm}$. To accelerate the dewetting and phase separation, samples are annealed at $170^{\circ} \mathrm{C}$ for three days in vacuum. PS-b-PMMA (50:50, $\mathrm{Mn}=$ $78.4 \mathrm{~kg} \cdot \mathrm{mol}^{-1}$, PDI $=1.09$ is used as a BCP. The PS-bPMMA powder is dissolved in PGMEA resulting in a
$1.5 \%(\mathrm{w} / \mathrm{w})$ solution. The BCP solution is spin-coated onto the brush layer to obtain a film with a uniform thickness. Afterward, the samples are annealed for $10 \mathrm{~min}$ at $230^{\circ}$ $\mathrm{C}$ in nitrogen in order to induce the BCP self-assembly.

\subsection{Atomic Force Microscopy}

Sample imaging of surface topography and surface material properties was obtained using an AFM apparatus (Dimension Icon, Bruker) operated in the PeakForce ${ }^{\mathrm{TM}}$ tapping mode. For topography imaging and PeakForce ${ }^{\mathrm{TM}}$ testing, standard tapping cantilevers (OTESPA, nominal radius of $7 \mathrm{~nm}$ ) and supersharp (SS) tip tapping cantilevers (TESP$\mathrm{SS}$, nominal radius of $3 \mathrm{~nm}$ ) are used. Both types of cantilevers have a nominal spring constant of $k=42 \mathrm{~N} / \mathrm{m}$. For samples in the modulus range ( $1 \mathrm{GPa}<E<20 \mathrm{GPa}$ ), stiffer cantilevers would guarantee better accuracy in modulus determination; however, in our experiments, they produced excessive damage to the samples. Applied force ranged between 5 and $300 \mathrm{nN}$; particularly, the force set point was adjusted for each contact event (tap) to obtain enough deformation for a reliable fit in the unload region (2 to $10 \mathrm{~nm}$ ) (see Fig. 1 for details). During measurements in PeakForce ${ }^{\mathrm{TM}}$ mode, the tip oscillates at a frequency $(2 \mathrm{kHz})$ far below the cantilever resonant frequency $(300 \mathrm{kHz})$. The vertical motion of the cantilever relies on deflection (force) signal for feedback, so that the surface position is acquired when the maximum cantilever deflection (the peak force) equals the force set point value. Interaction peak force and material property information is collected for each individual tap. By using the calibration of the optical lever sensitivity, the cantilever spring constant, ${ }^{13}$ and the tip radius, the force versus distance curves provide quantitative information of elastic modulus, adhesion force, sample deformation, and dissipated energy.

\subsection{Data Analysis}

As a reference, in Fig. 1, we report one force versus distance curve obtained with a single approach (red line) and withdrawal (blue line) on a PS sample. The various regions

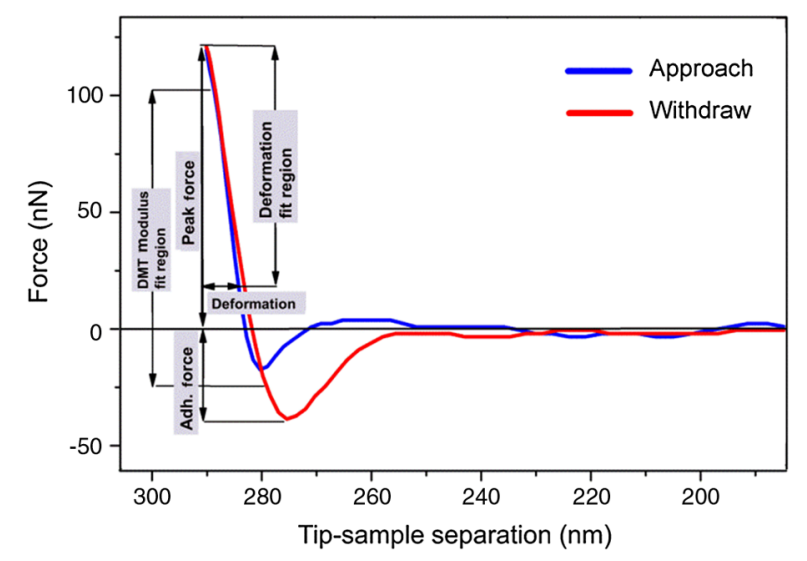

Fig. 1 Typical indentation curve obtained with a cantilever of nominal stiffness $k=42 \mathrm{~N} / \mathrm{m}$ upon a polystyrene (PS) reference sample of $E \approx 2.7 \mathrm{GPa}$. The deformation is defined as the distance from the base of the deformation fit region position to the peak interaction force position. (The deformation fit region is $85 \%$ of full deformation.) The deformation during the experiments has been kept above $2 \mathrm{~nm}$. The portion of the withdraw curve used for the modulus fit is also indicated (typically $70 \%$ of the unload force curve). 
marked indicate the regions and parameters taken into consideration during the calculation of mechanical properties. To determine the elastic modulus of the sample, the curve is fitted with the Derjaguin-Muller-Toporov (DMT) model, ${ }^{14}$ applicable for systems with low adhesion and small tip radii, using a portion of the unload curve as the fit region. The DMT model is a modified Hertzian model, which also takes into account the adhesive forces. In fact, as the indenter retracts, it is possible to measure the adhesiveness between the tip and the surface ${ }^{15}$ (Fig. 1). The reduced modulus $E_{r}$ is given by

$F=\frac{4}{3} E_{r} \sqrt{R d^{3}}+F_{a d h}=k z$,

where $F$ is the force, $R$ is the tip radius, $d$ is the deformation, $F_{a d h}$ is the maximum adhesion force, and $z$ is the vertical displacement.

In the present work, we will always refer to the reduced modulus $E_{r}$. The relation between $E_{r}$ and the sample modulus $E_{S}$ is

$E_{r}=\left[\frac{1-\nu_{t}^{2}}{E_{t}}+\frac{1-\nu_{s}^{2}}{E_{s}}\right]^{-1}$,

where $\nu_{t}$ and $E_{t}$ are the Poisson's ratio and elastic modulus of the tip, and $\nu_{s}$ and $E_{s}$ are the Poisson's ratio and elastic modulus of the sample. Assuming that the tip modulus, $E_{t}$, is much larger than the sample modulus $\left(E_{t} \approx 130 \mathrm{GPa}\right)$, we can neglect the first addendum. If $\nu_{s}$ is equal to 0.33 ( $1 \mathrm{GPa}<E_{s}<10 \mathrm{GPa}$ ), the relation between the reduced and sample modulus becomes $E_{s}=0.89 E_{r}$.

The tip radius was calculated by an indirect method where the radius was adjusted to achieve the correct value of a sample with known elastic modulus (PS reference film, $E=2.7 \mathrm{GPa}$ ). The topography images were subjected to a second-order polynomial flattening algorithm to correct surface tilt and bow effects. Some of the modulus images obtained at $1 \times 1 \mu \mathrm{m}^{2}$ scan size were subjected to equalized flattening (order 2) and FFT high pass filter smoothing in order to remove noise induced by optical interferences in the detection system which would lead to fluctuations in the baseline values of the force curves.

\section{Results and Discussion}

\subsection{Formation of Different Phases of Polystyrene and Poly(Methyl Methacrylate) Homopolymers}

As a first study, three kinds of samples were prepared (cast) using the same PS brush as a substrate: (1) PS homopolymer, (2) PMMA homopolymer, and (3) PS/PMMA blend. The films, as casted and upon the quick evaporation of the solvent, leave homogeneous layers that do not present holes or droplets. This is an indication that for our polymers at ambient temperature, interfacial interaction rules the wetting behavior. ${ }^{13,14}$ After induced dewetting (i.e., accelerated by an annealing of three days at $170^{\circ} \mathrm{C}$ ), the final morphology of the surface consisted of homopolymer semispherical droplets of diameters between 0.5 and $3 \mu \mathrm{m}$ upon the PS brush layer (Fig. 2).

The size and geometry of the droplets allows us to verify the critical film height that induces the so-called "mechanical double-layer effect" 16 or, in other words, the minimum thickness of polymer that we can probe by PeakForce ${ }^{\mathrm{TM}}$ for a given indentation, without measuring the influence of the stiff substrate underneath. For a semispherical PS droplet, the reduced modulus image [Fig. 2(a)] clearly shows a brighter ring surrounding the drop marking the transition region between the beginning and the end of the doublelayer effect.

The reduced modulus values for both PS and PMMA homopolymers measured by probing the center of the droplet (thickness $>500 \mathrm{~nm}$ ) are in agreement with the values reported for conventional AFM experiments at similar indentation depths. ${ }^{17}$ Although slightly smaller than 3.9 GPa, which is the value reported for bulk PS with similar $\mathrm{Mn},{ }^{18} \mathrm{PS}$ droplets provide a value of $E_{r}=2.6 \pm 0.3 \mathrm{GPa}$.
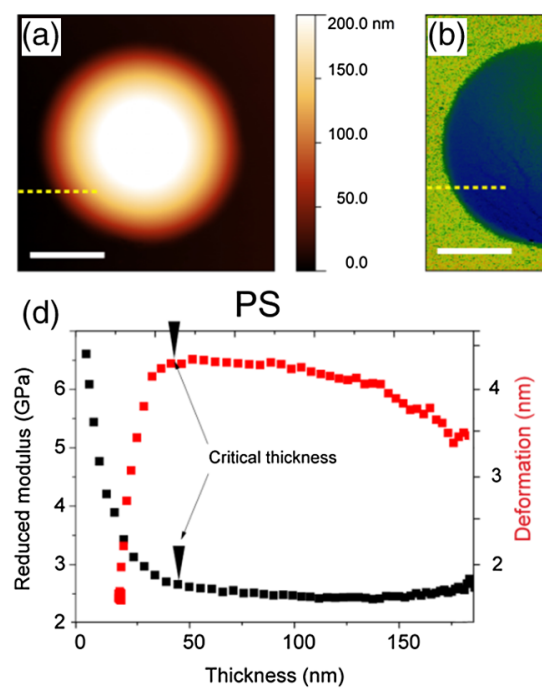
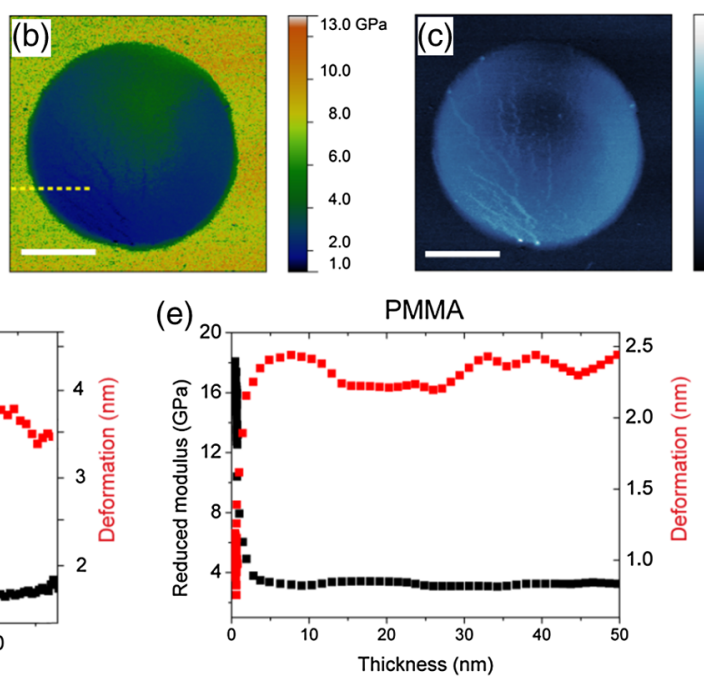

Fig. 2 (a) Atomic force microscopy (AFM) topography, (b) reduced modulus, and (c) deformation maps of PS homopolymer drops on hydroxyl-terminated polystyrene (PS-OH) after dewetting. Scale bar is $1 \mu \mathrm{m}$. In (d), Derjaguin-Muller-Toporov (DMT) modulus and deformation plotted versus sample thickness. Data taken from the profile marked with a yellow dashed line in (a) and (b). The black arrows point to the end of the transition region between the beginning and the end of the double layer effect. In (e), DMT modulus and deformation is plotted versus sample thickness for a poly(methyl methacrylate) (PMMA) droplet. 

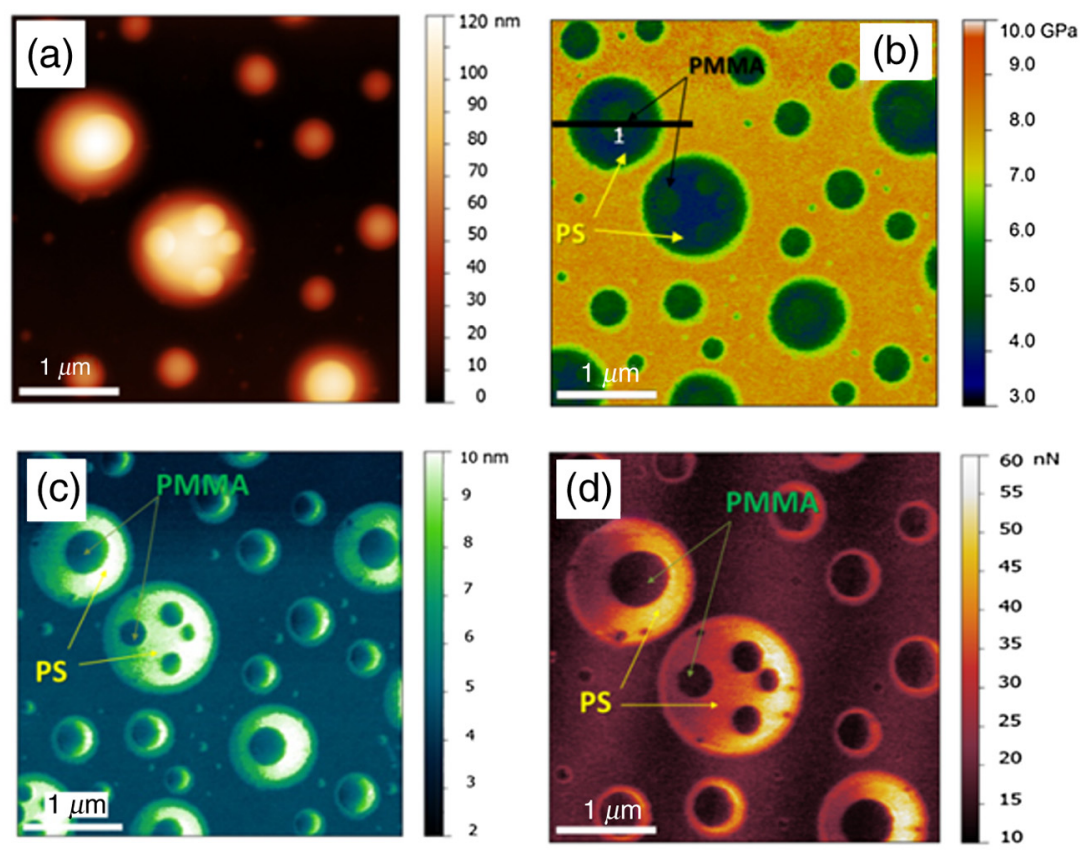

(e)

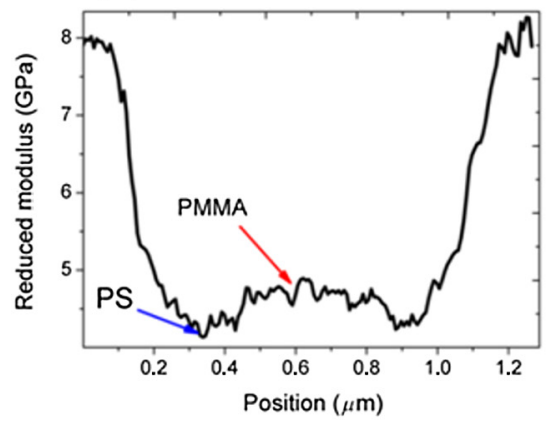

(f)

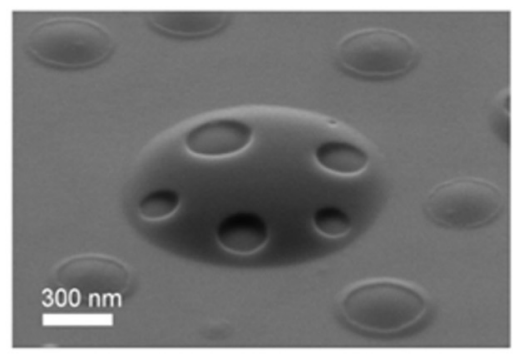

Fig. 3 (a) AFM topography, (b) reduced modulus, (c) deformation, and (d) adhesion maps of PS/PMMA homopolymer blend on PS-OH after dewetting. In (e), profile 1 is reported in detail. Difference in modulus map between the two components is better visualized in deformation and adhesion maps, components are indicated by arrows. In (f), SEM image of the blend after PMMA removal by oxygen plasma is shown.

Similar tests on dewetted PMMA droplets result in a value of $E_{r}=3.3 \pm 0.5 \mathrm{GPa}$. Values are calculated from a Gaussian fit of the relevant portion of the modulus values' distribution over the whole image [Fig. 2(b)]. Rather than specifically determining the surface elastic modulus of our samples with statistical relevance, the aim is to estimate the minimum polymer film thickness that can be probed under present experimental conditions, as follows.

We plot a portion of the data of reduced modulus and deformation versus height as extracted from one image profile [Fig. 2(d)], identifying what height value yields constant values of $E_{r}$. As indicated by the black arrow in the plot, PS samples thicker than $48 \mathrm{~nm}$ return a near bulk-like value of $E_{r}$. Consistently, the threshold value at which the mechanical double-layer effect becomes negligible for a PS thin layer that is indented in a similar way $(\approx 4 \mathrm{~nm}$ of indentation $)$ is $\sim 48 \mathrm{~nm}$. Similar data for a PMMA droplet under the same applied force $(40 \mathrm{nN})$ are plotted in Fig. 2(e). Results show a much lower threshold (thinner) where the end of the doublelayered effect is estimated to be $\sim 5 \mathrm{~nm}$. This means that PMMA elastic behavior is less influenced by the substrate.

As already mentioned, the mechanical properties of the surface of a material (e.g., testing thin films) may vary consistently from the bulk values. Apart from the influence of the rigid substrate, there are other phenomena that influence indentation experiments performed with an AFM. ${ }^{10,19}$ Polymer thin film's glass transition temperature may increase or decrease with respect to bulk values depending on the film thickness and the interaction with the supporting substrate. ${ }^{20} \mathrm{~A}$ (softer) liquid-like layer is probably present at the polymer-air interface, ${ }^{21}$ even if its influence could be neglected due to the compression exerted by the tip during indentation. Moreover, end chains with lower surface energy tend to migrate to the surface of glassy polymers. ${ }^{22}$ These factors lead to difficulties in the quantitative interpretation of the obtained modulus values. Nevertheless, PeakForce ${ }^{\mathrm{TM}}$ technique allows sensing compositional differences some nanometers below the surface, while standard tapping phase imaging senses just the surface.

PS/PMMA blends after dewetting present droplet morphologies similar to the single components (Fig. 3). PS larger droplets incorporate PMMA smaller droplets. Considering the $E_{r}$ map [Fig. 3(b)], harder areas corresponding to PMMA areas are apparent. In the surface elastic modulus profile shown in Fig. 3(e), harder PMMA, $E_{r} \approx 5 \mathrm{GPa}$, is embedded into the softer PS matrix surrounding it. From 
indentation and adhesion maps [Figs. 3(c) and 3(d)], it is possible to better distinguish the two blend components. For a constant force set point, the indentation is smaller on the PMMA domains than in the PS regions because the elastic modulus of PMMA is higher than that of PS. Moreover, the adhesion map suggests that a PMMA layer is covering the brush, as confirmed by some additional measurements made on samples exposed to oxygen plasma in order to selectively eliminate PMMA. As expected, the adhesion force decreases with the increase of elasticity modulus and indentation hardness.

The sample covered with randomly aligned oriented PSb-PMMA BCP consisted of a $36 \mathrm{~nm}$ thick film (as measured by AFM), i.e., a few nanometers below the probing threshold for PS, although the presence of a stiffer phase (PMMA) should have compensated such an effect. The random alignment is shown in Fig. 4(a) and presents the expected pitch of $42 \mathrm{~nm}$ (as determined by the distance between the edges of two features with the same composition).

In the following experiments, we imaged the BCP surface with a standard silicon tip (OTESPA) and with SS tips. The latter allows to reduce the contact radius to $a=\sqrt{R d}$, assuming that OTESPA tips have a nominal radius of $6 \mathrm{~nm}$, while SS tips have a nominal radius of $3 \mathrm{~nm}$. For brevity, we only report the images obtained with SS tips [Figs. 4(b)-4(d)], while $E$ values obtained from $1 \times 1 \mu \mathrm{m}^{2}$ scans for different tips/indentations are summarized in Fig. 4(h), showing how the stiffening effect of the substrate tends to appear for larger indentation $(>4 \mathrm{~nm})$. Figure $4(\mathrm{~g})$ reports $E_{r}$ values' distribution extracted from Fig. 4(c), including the suggested Gaussian fits of the two components; the two components are not fully separated in the histogram. Actually, better separation occurs in the deformation channel, leading to the conclusion that SS tips are performing in a way similar to standard OTESPA in terms of lateral resolution. It is clear that in this specific experiment with the SS tip, the module of both phases is slightly overestimated due to excessive deformation [blue arrows in Fig. 4(h)] even if other channels (deformation and adhesion) present enough contrast to distinguish the two segregated phases [Figs. 4(d) and 4(e)]. Harder PMMA $\left(E^{*} \approx 5.5 \mathrm{GPa}\right)$ is represented in light green in Fig. 4(c) and softer PS has an $E^{*} \approx 4.3 \mathrm{GPa}$. However, it is worth mentioning that contrast switches in the deformation and adhesion maps [Figs. 4(d) and 4(e)]. As expected, PS reveals a higher adhesion $(\approx 12 \mathrm{nN})$ than the PMMA phase $(\approx 8 \mathrm{nN})$. Interestingly, switching to a smaller contact radius but keeping deformation around $2 \mathrm{~nm}$ [circled points in Fig. 4(h)], the obtained values of $E_{r}$ match what is measured with larger tip radii, i.e., applying larger loads, indicating $E^{*}$ values of $4.10 \pm 0.34$ and $3.25 \pm 0.22 \mathrm{GPa}$ for PMMA and PS phases, respectively. Sharper tips can accurately image the $\mathrm{BCP}$, reducing the force needed to reach a sufficient indentation depth. This aspect is particularly relevant because when the ratio between the contact radius and film thickness reaches close to 0.1 (interfacial zone), any fitting becomes particularly challenging. ${ }^{8-1623,24}$

For indentation between 2 and $4 \mathrm{~nm}$ [circled points in Fig. 4(g)], $E$ values are not affected by the presence of the substrate. Anyhow, the surface portion of the polymer probed presents interfacial behaviors that do not allow us to uniquely link $E$ values obtained with the physical properties of the two phases. More studies will be needed in order to specifically distinguish the dominating factors.

\section{Conclusions}

In conclusion, we used AFM nanomechanical characterization by PeakForce ${ }^{\mathrm{TM}}$ tapping in order to probe PS/PMMA BCP samples, which are of great interest for further technological developments. We showed that the PeakForce ${ }^{\mathrm{TM}}$ technique can be used to measure nanomechanical properties, such as surface elastic modulus and adhesion forces, on samples with a critical thicknesses $<50 \mathrm{~nm}$, that is samples that cannot be probed by standard indentation experiments. Through the methodology proposed, it is possible to acquire a large amount of data (force curves) at each scan, and due to the small volume of interaction $\left(\right.$ few $\mathrm{nm}^{3}$ ), these data are linked to the different BCP nanodomains. In the case of PS/PMMA based BCPs, the values of $E_{r}$ obtained for BCP film are slightly higher than bulk PS and PMMA confirming previous experimental evidences. Once the optimal

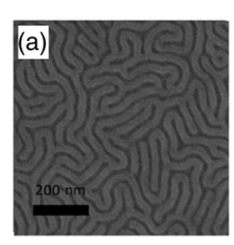

(f)

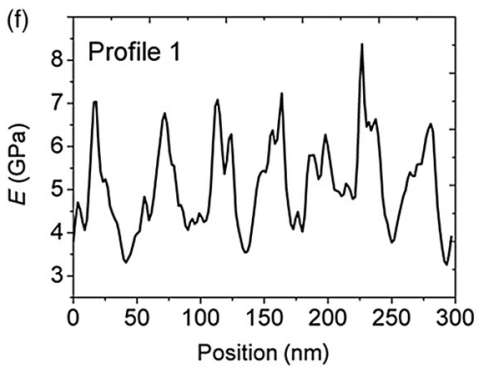

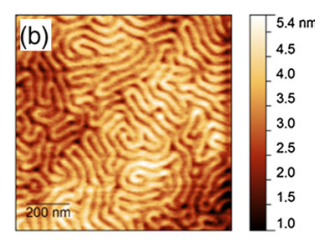

(g)

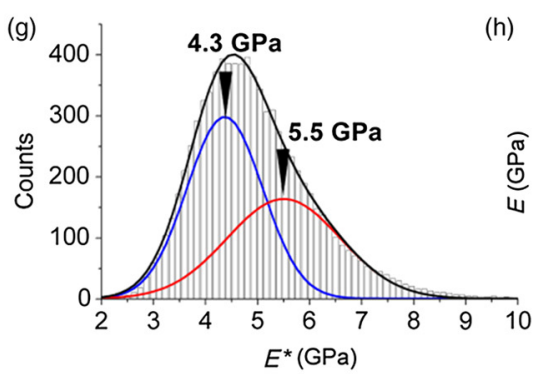

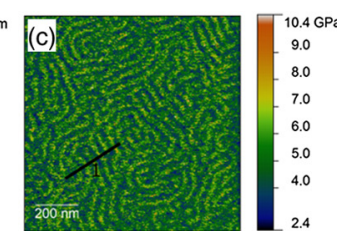

$E^{*}(\mathrm{GPa})$
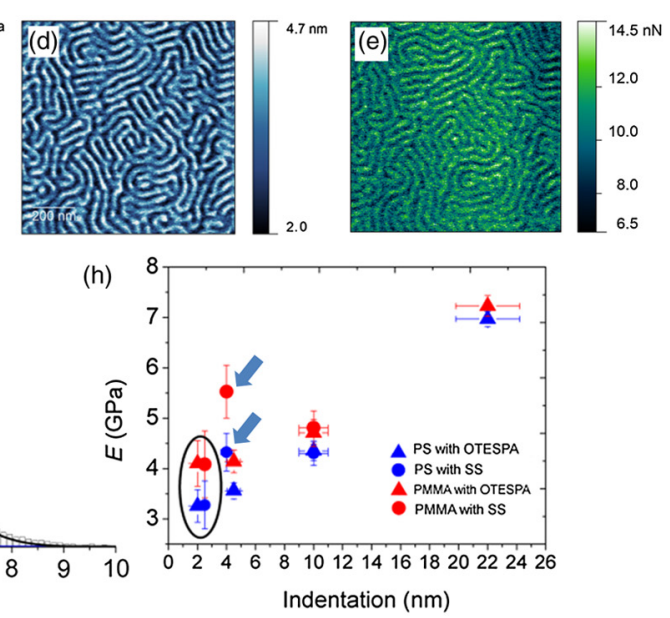

Fig. 4 (a) SEM image, (b) AFM topography, (c) reduced modulus, (d) deformation, and (e) adhesion maps of a randomly oriented PS- $b$-PMMA block copolymer thin film. SEM images are taken after removing the PMMA block by oxygen plasma. (f) Reduced modulus values along profile 1. (g) Reduced modulus distribution with a two Gaussian suggested fit to identify $E^{*}$ of each phase. Similar fits led to compose the graphic in (h); note that indentation is given as an average indentation on the whole scan and not of a single polymer. The images in (b) to (d) were obtained with an ultrasharp tip. 
indentation conditions are identified, PeakForce ${ }^{\mathrm{TM}}$ with standard cantilevers can resolve BCP structures with a pitch down to $42 \mathrm{~nm}$ and further improvement can be reached by adopting smaller radius indenters, such as SS tips.

\section{Acknowledgments}

All authors have revised and given approval to the final version of this paper. This work was partially funded by the projects SNM (FP7-ICT-2011-8) and FORCE-for-FUTURE (CSD2010-00024).

\section{References}

1. S. O. Kim et al., "Epitaxial self-assembly of block copolymers on lithographically defined nanopatterned substrates," Nature 424, 411-414 (2003).

2. M. P. Stoykovich et al., "Directed assembly of block copolymer blends into nonregular device-oriented structures," Science 308, 1442-1446 (2005).

3. M. Fernández-Regúlez et al., "Sub-10 nm resistless nanolithography for directed self-assembly of block copolymers," ACS Appl. Mater. Interfaces 6, 21596-21602 (2014).

4. F. Bates, "Block copolymer thermodynamics: theory and experiment," Annu. Rev. Phys. Chem. 41, 525-557 (1990).

5. D. Borah et al., "The morphology of ordered block copolymer patterns as probed by high resolution imaging," Nanomater. Nanotechnol. 4-25 (2014).

6. G. Krämer et al., "Study of morphology and mechanical properties of polystyrene-polybutadiene blends with nanometre resolution using AFM and force-distance curves," Eur. Polym. J. 55, 123-134 (2014).

7. M. Sababi et al., "Structural and nanomechanical properties of paperboard coatings studied by peak force tapping atomic force microscopy," ACS Appl. Mater. Interfaces 4, 5534-5541 (2012).

8. T. J. Young et al., "The use of the PeakForce TM quantitative nanomechanical mapping AFM-based method for high-resolution Young's modulus measurement of polymers," Meas. Sci. Technol. 22, 125703 (2011).

9. B. Cappella and D. Silbernagl, "Nanomechanical properties of polymer thin films measured by force-distance curves," Thin Solid Films 516, 1952-1960 (2008).

10. B. Du et al., "Study of elastic modulus and yield strength of polymer thin films using atomic force microscopy," Langmuir 17, 3286-3291 (2001).

11. P. Trtik, J. Kaufmann, and U. Volz, "On the use of peak-force tapping atomic force microscopy for quantification of the local elastic modulus in hardened cement paste," Cem. Concr. Res. 42, 215-221 (2012).

12. E. T. Herruzo, A. P. Perrino, and R. Garcia, "Fast nanomechanical spectroscopy of soft matter," Nat. Commun. 5, 3126 (2014).

13. J. E. Sader, J. W. M. Chon, and P. Mulvaney, "Calibration of rectangular atomic force microscope cantilevers," Rev. Sci. Instrum. 70, 3967 (1999).

14. B. Cappella and G. Dietler, "Force-distance curves by atomic force microscopy," Surf. Sci. Rep. 34, 1-104 (1999).

15. U. D. Cakmak, T. Schöberl, and Z. Major, "Nanoindentation of polymers," Meccanica 47, 707-718 (2012).

16. B. Cappella and D. Silbernagl, "Nanomechanical properties of mechanical double-layers: a novel semiempirical analysis," Langmuir $\mathbf{2 3}$, 10779-10787 (2007).

17. K. Miyake, N. Satomi, and S. Sasaki, "Elastic modulus of polystyrene film from near surface to bulk measured by nanoindentation using atomic force microscopy," Appl. Phys. Lett. 89, 031925 (2006).

18. J. M. Torres, C. M. Stafford, and B. D. Vogt, "Impact of molecular mass on the elastic modulus of thin polystyrene films," Polymer $\mathbf{5 1}$, 4211-4217 (2010).

19. C. M. Stafford et al., "A buckling-based metrology for measuring the elastic moduli of polymeric thin films," Nat. Mater. 3, 545-550 (2004).

20. W. E. Wallace, J. H. Van Zanten, and W. L. Wu, "Influence of an impenetrable interface on a polymer glass-transition temperature," Phys. Rev. E 52 (1995).

21. J. L. Keddie, R. A. L. Jones, and R. A. Corey, "Size-dependent depression of the glass transition temperature in polymer films," Europhys. Lett. 27, 59-64 (1994).

22. K. Tanaka et al., "Effect of chain end chemistry on surface molecular motion of polystyrene films," Macromolecules 31, 5148-5149 (1998).
23. B. Cappella, "Mechanical properties and adhesion of a micro structured polymer blend," Polymers 3, 1091-1106 (2011).

24. R. Seemann et al., "Dynamics and structure formation in thin polymer melt films," J. Phys. Condens. Matter 17, S267-S290 (2005).

Matteo Lorenzoni is a postdoc researcher at the Barcelona Institute of Microelectronics. He received his MS degree in chemistry from the University of Genoa in 2002 and his PhD in nanoscience from the Italian Institute of Technology in 2014, where he presented a PhD defense centered on recent advances in nanofabrication through AFM. His current research interests include atomic force microscopy, nanolithography, and block copolymer self-assembly.

Laura Evangelio is a $\mathrm{PhD}$ candidate shared by the Barcelona Institute of Microelectronics (IMB-CNM) and the Institut Català de Nanociència i Nanotecnologia. She graduated in chemical engineering at the Universitat de Barcelona (UB) in 2011 and got her master's degree at the Autonomous University of Barcelona. She is trained in the use of clean room facilities and nanotechnology fabrication methods. Her present research focuses on the creation of guiding patterns for block-copolymer self-assembly.

Célia Nicolet is an R\&D engineer at Arkema. In 2005, she started an engineering course at the National School of Chemistry and Physics of Bordeaux. During this training, she had the opportunity to do several internships, including one in the Netherlands in the laboratory of R\&D Nuplex, a resin for paints manufacturing company. During the postdoctoral year, she worked on the development of resins for nanolithography in partnership with Arkema, and now she is an R\&D engineer.

Christophe Navarro is a senior scientist at Arkema Inc., a global chemical company and France's leading chemicals producer. He has been in charge of various laboratories since 1996. His specialties include controlled polymerization, block-copolymer synthesis, and impact modifiers. He is a project leader of various projects for Corporate and Functional Additives B.U. and is participating in the European project Computational Lithography for Directed SelfAssembly: Materials, Models, and Processes.

Alvaro San Paulo is a senior scientist at the Madrid Microelectronic Institute (IMM-CSIC). He has published more than 40 papers on manifold topics such as atomic force microscopy, silicon nanowires, nanomechanical sensors, nanoelectromechanical systems, and nanofabrication. He is now participating in the Force-For-Future project that proposes an integrated approach to develop a new paradigm in force microscopy that will overcome some of the current weaknesses of probe microscopy.

Gemma Rius is an assistant professor at NITech, Japan. She started her research on nanotechnology at the Institute of Microelectronics of Barcelona (Spain) and became an expert in nanopatterning and Si nanofabrication. She has been working on nanocarbon synthesis and electronic applications. She is also involved in advanced AFM techniques for characterization and manipulation of surfaces and nanomaterials. She is author of more than 50 articles and several book chapters/reviews on carbon nanomaterials and nanotechnology.

Francesc Pérez-Murano is a research professor at the Microelectronics Institute of Barcelona (IMB-CNM, CSIC). He is head of the nanofabrication and functional properties of nanostructures group. He graduated from the Autonomous University of Barcelona (UAB) with a degree in physics, and later, he received a $\mathrm{PhD}$ in science for the same university in 1989. He has been associate professor in the Electronics Engineering Department at UAB from 1995 to 2001, where he became vice director of the engineering school in 2000. In 2001, he joined IMB-CNM, CSIC, being promoted to research professor in 2007. 